\title{
Plasmon dispersion in molecular solids: Picene and potassium-doped picene
}

\author{
Pierluigi Cudazzo, ${ }^{1}$ Matteo Gatti, ${ }^{1}$ Friedrich Roth, ${ }^{2}$ Benjamin Mahns, ${ }^{2}$ Martin Knupfer,${ }^{2}$ and Angel Rubio ${ }^{1,3}$ \\ ${ }^{1}$ Nano-Bio Spectroscopy group and ETSF Scientific Development Centre, Departamento Física de Materiales, Universidad del País Vasco, \\ Centro de Física de Materiales CSIC-UPVIEHU-MPC and DIPC, Av. Tolosa 72, ES-20018 San Sebastián, Spain \\ ${ }^{2}$ IFW Dresden, P.O. Box 270116, DE-01171 Dresden, Germany \\ ${ }^{3}$ Fritz-Haber-Institut der Max-Planck-Gesellschaft, Theory Department, Faradayweg 4-6, DE-14195 Berlin-Dahlem, Germany
}

(Received 16 June 2011; published 14 October 2011)

\begin{abstract}
We investigate the dynamic response of pristine and potassium-doped picene, the first example of a new family of organic molecular superconductors, by combining first-principles calculations and state-of-the-art experimental tools. We find that charge-carrier plasmons in $\mathrm{K}_{3}$ picene have a negative or almost negligible dispersion, which deviates from the traditional picture of metals based on the homogeneous electron gas. We show how this finding is the result of the competition between metallicity and electronic localization on the molecular units. Conduction electrons alone give rise to the negative dispersion, which is reduced by molecular polarization and crystal local-field effects. This analysis allows us to obtain a general picture of the plasmon dispersion in metallic molecular crystals.
\end{abstract}

DOI: $10.1103 /$ PhysRevB.84.155118

PACS number(s): 79.20.Uv, 71.45.Gm, 77.84.Jd, 74.70.Kn

\section{INTRODUCTION}

Organic molecular solids have attracted considerable interest for both fundamental reasons and possible technological applications. In fact, they are very promising materials for a wide range of applications, ${ }^{1}$ as their properties can be efficiently tailored for their use in (opto)electronic devices such as field-effect transistors, ${ }^{2}$ light-emitting diodes, ${ }^{3,4}$ photovoltaic cells, ${ }^{5,6}$ etc. Their building blocks are molecular units held together by weak van der Waals forces. The constituents thus preserve their molecular character, giving rise to nondispersive bands with $\sigma$ and $\pi$ character. Therefore, from the fundamental point of view, organic molecular solids represent a playground where it is possible to bridge molecular and solid-state physics and where both strong electron-phonon and electron-electron interactions in the narrow bands are expected to be fully at work. The rich variety of electronic properties that is possible to obtain in this class of materials in fact emerges as result of the competition between charge localization on a single molecule (where strong covalent bonds are active) and intermolecular tunneling of $\pi$ electrons (whence metallicity), which is facilitated by the large extension of the molecular units. When the former prevails, molecular solids are semiconductors or insulators, with a gap opening between bands deriving from bonding $\pi$ and antibonding $\pi^{*}$ molecular states. Doping with alkali metals introduces new electrons that occupy the empty $\pi^{*}$ states. Thus molecular crystals can become metals or even superconductors with high critical temperatures, as in the case of fullerides. ${ }^{7-9}$ Very recently, the discovery of superconductivity in potassium-doped picene ${ }^{10}$ has opened a new path within an unanticipated group of molecular superconductors. In fact, superconductivity has been then reported also in other doped polycyclic aromatic hydrocarbons, like phenanthrene ${ }^{11}$ and coronene. $^{12}$

Given the many competing excitations, the many open questions in these materials, like the mechanism of charge transport (incoherent phonon-assisted hopping or coherent band transport) ${ }^{13}$ or the (unconventional) superconducting pairing, ${ }^{9,14}$ still represent a major challenge for fundamental research. An essential contribution to clarify these unsolved questions can be obtained by studying the elementary excitations that characterize the electronic properties of these materials. In the present work, we therefore investigate the dynamic response of both pristine and doped picene, as measured by electron-energy loss spectroscopy (EELS), combining first-principles calculations and state-of-the-art experimental tools. In particular, we demonstrate how the competition between metallicity and electronic localization in $\mathrm{K}_{3}$ picene gives rise to plasmons with negative or almost negligible dispersion. We further discuss that this is a behavior peculiar to doped molecular crystals in general, and which contrasts the positive plasmon dispersion in the homogeneous electron gas (HEG), which is often taken as reference in the studies of electronic response.

Picene molecules consist of five benzene rings joined in an armchair manner. They crystallize in a monoclinc structure, where the primitive cell contains two inequivalent molecules arranged in a herringbone pattern in the $a b$ plane so that the $c$ axis is nearly parallel to the molecular main axis, while $a$ and $b$ axes are out of the molecular plane. In the solid phase the bonding-antibonding character of the molecular orbitals causes a band-gap opening of $4 \mathrm{eV}$ between $\pi$ and $\pi^{*}$ bands. ${ }^{15}$ In $\mathrm{K}_{3}$ picene, the six additional electrons from potassium atoms occupy the lowest four overlapping $\pi^{*}$ bands and the system becomes metallic. ${ }^{16,17}$

\section{EXPERIMENTAL AND COMPUTATIONAL METHODS}

For our experiments using electron energy-loss spectroscopy (EELS) in transmission, we have prepared picene films with a thickness of about $100 \mathrm{~nm}$, by thermal evaporation under high vacuum onto single crystalline $\mathrm{KBr}$ substrates kept at room temperature. Thereby, two deposition rates, $0.2 \mathrm{~nm} / \mathrm{min}$ and $4 \mathrm{~nm} / \mathrm{min}$, have been chosen in order to obtain films with different crystalline texture. These picene films were floated off in distilled water, mounted onto standard electron microscopy grids and transferred into the spectrometer. Prior to the EELS measurements, the films were characterized in situ using electron diffraction. All observed diffraction peaks were 
consistent with the crystal structure of picene. ${ }^{18}$ Moreover, the diffraction spectra revealed a pronounced texture, whereas the films grown with a deposition rate of $0.2 \mathrm{~nm} / \mathrm{min}$ showed a strong preference of crystallites with their $a b$ plane parallel to the film surface, while those films grown with $4 \mathrm{~nm} / \mathrm{min}$ showed a substantial amount of crystallites with their $c$ axis in the film surface. All electron diffraction studies and loss function measurements were carried out using the $172 \mathrm{keV}$ spectrometer described in detail elsewhere. ${ }^{19}$ We note that at this high primary beam energy only singlet excitations are possible. The energy and momentum resolution were chosen to be $85 \mathrm{meV}$ and $0.03 \AA^{-1}$, respectively. We have measured the loss function $-\operatorname{Im} \epsilon_{M}^{-1}(\mathbf{Q}, \omega)$, for a momentum transfer $\mathbf{Q}$ parallel to the film surface $\left[\epsilon_{M}(\mathbf{Q}, \omega)\right.$ is the macroscopic dielectric function]. Potassium was added in several steps by evaporation from commercial SAES (SAES GETTERS S.p.A., Italy) getter source under ultrahigh vacuum conditions (base pressure lower than $10^{-10}$ mbar) until a doping level of about $\mathrm{K}_{3}$ picene was achieved. In detail, in each doping step, the sample was exposed to potassium for 5 min, the current through the SAES getter source was $6 \AA$ and the distance to the sample was about $30 \mathrm{~mm}$. During potassium addition, the film was kept at room temperature. Postannealing of the films at $440 \mathrm{~K}$ for several hours did not lead to changes in the doping level, which demonstrates that potassium diffusion at room temperature is sufficient to achieve a homogeneous distribution in the film (see also Ref. 28).

To simulate the experimental spectra, we have calculated the loss function $-\operatorname{Im} \epsilon_{M}^{-1}(\mathbf{Q}, \omega)$, which is obtained from the diagonal element of the inverse of the microscopic dielectric function $\epsilon$ as 20,21

$$
\epsilon_{M}(\mathbf{Q}, \omega)=\frac{1}{\epsilon_{\mathbf{G}, \mathbf{G}}^{-1}(\mathbf{q}, \omega)}
$$

Here, $\mathbf{Q}=\mathbf{q}+\mathbf{G}$, where $\mathbf{q}$ belongs to the first Brillouin zone and $\mathbf{G}$ is a vector of the reciprocal lattice. In a solid, the microscopic dielectric function $\epsilon$ is a matrix in the reciprocal-lattice vectors $\mathbf{G}$ and $\mathbf{G}^{\prime}$. Off-diagonal elements of $\epsilon$ are related to inhomogeneities in the induced charge densities that give rise to the crystal local-field effects (LFE). When LFE are neglected, Eq. (1) simplifies to $\epsilon_{M}(\mathbf{Q}, \omega)=$ $\epsilon_{\mathbf{G}, \mathbf{G}}(\mathbf{q}, \omega)$. Our calculations are based on density-functional theory (DFT) in the local-density approximation (LDA) implemented in a pseudopotential plane-wave framework. ${ }^{22,23}$ We have optimized the crystal structure of pristine picene starting from the experimental atomic positions. ${ }^{18}$ The crystal structure of potassium-intercalated picene instead is not fully known experimentally. We have therefore used the crystal structure calculated in Ref. 16, in which potassium atoms are in intralayer positions. We have also adopted the crystal structure proposed in Ref. 24. But the worse agreement obtained with respect to the experimental spectra seems to rule out this alternative structure. We have calculated $\epsilon$ in the random-phase approximation (RPA) using 360 LDA bands in a $8 \times 8 \times 6$ Monkhorst-Pack grid of $\mathbf{k}$ points. To obtain $\epsilon^{-1}$ in Eq. (1) taking into account LFE, we have inverted a matrix of rank $321 \mathbf{G}$ vectors.

\section{PRISTINE PICENE}

To gain insight into the effects on the plasmon dispersion of the electron localization on the molecular units, we first consider pristine picene. In the calculated loss functions with momentum transfers along the three reciprocal axes $a^{*}, b^{*}$, and $c^{*}$, we can identify five sharp features [(A) to (E) in Figs. 1(a), 1 (b) and 2(a)] in the energy range below $10 \mathrm{eV}$. This is verified by recognizing the same structures also in the experimental spectra [Figs. 1(c) and 2(c)]. As discussed in Ref. 15, only the peak $(\mathrm{E})$ is due to a plasmon excitation, while all the other features are due to interband transitions. The differences between theory and experiment are due to two reasons: (i) the theoretical spectra display a redshift in the peak positions, as
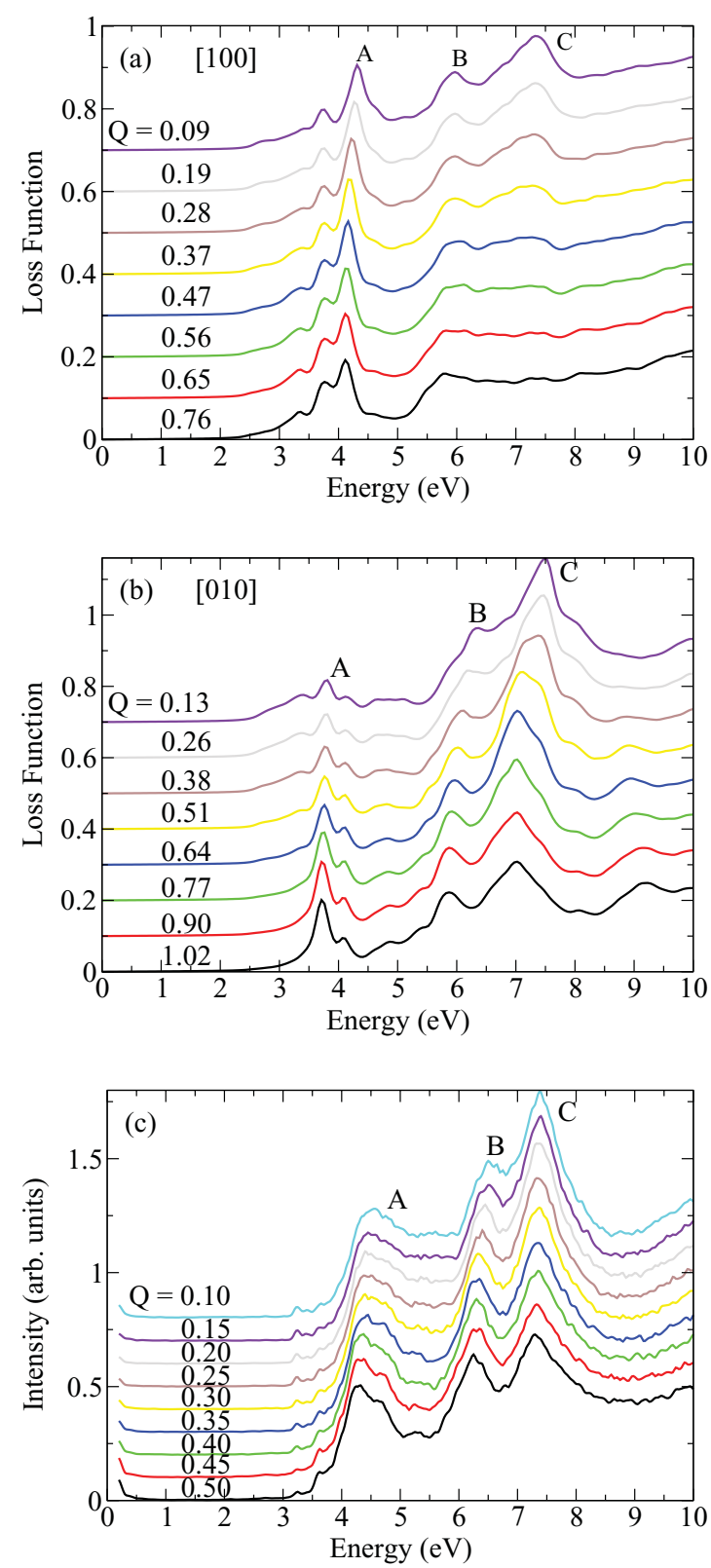

FIG. 1. (Color online) Calculated loss function of picene for momentum transfer $\mathbf{Q}$ (in $\AA^{-1}$ units) along the $a^{*}$ (a) and $b^{*}$ (b) axes, respectively, and experimental EELS for samples with preferential orientation in the $a b$ plane (c). 

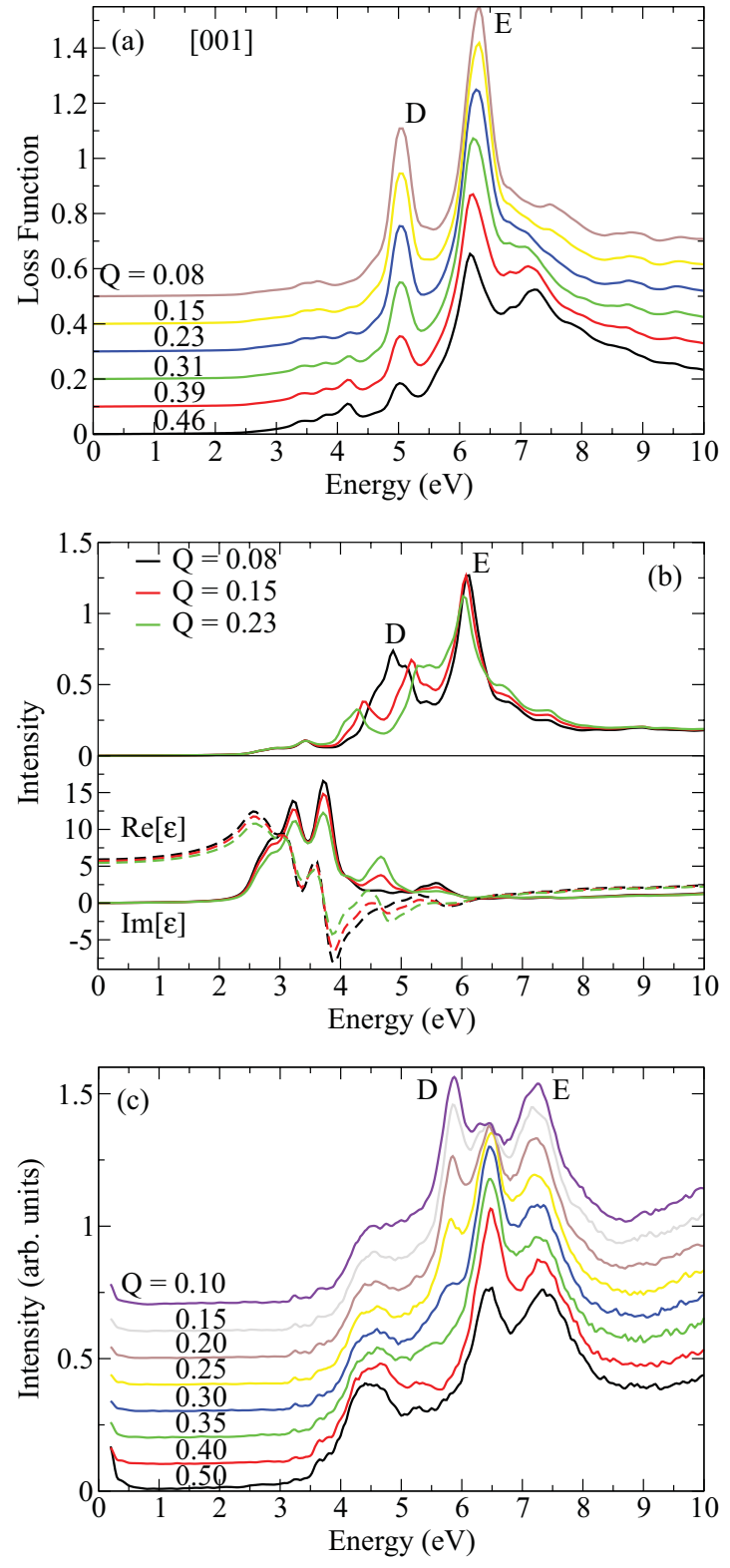

FIG. 2. (Color online) Calculated loss function of picene for momentum transfer $\mathbf{Q}$ (in $\AA^{-1}$ units) along the $c^{*}$ axes (a) and experimental EELS for samples with preferential orientation along the $c$ axis (c). (b) Loss function (upper panel) and real and imaginary part of the dielectric function (bottom panel) for $\mathbf{Q}$ along $c^{*}$ calculated without LFE.

a consequence of the fact that LDA band-gap underestimation is larger than the electron-hole binding energy, ${ }^{15}$ and (ii) in the experimental samples, a mixture of crystallite directions is always present, which is the reason why the structures of the theoretical spectra along the different axes appear mixed in the experimental spectra.

The various excitations in the energy loss spectra still keep trace of their molecular origin. Picene molecule belongs to the $C_{2 v}$ point group. Thus, for symmetry reasons, the only allowed electronic transitions in the dipole approximation are $\pi \rightarrow \pi^{*}$ and $\sigma \rightarrow \sigma^{*}$ for polarization directions belonging to the plane of the molecule and $\pi \rightarrow \sigma^{*}$ and $\sigma \rightarrow \pi^{*}$ for the direction perpendicular to the plane. In solid picene, for the small overlap between wave functions localized on different sites, this picture is approximately still valid. In LDA, $\pi\left(\pi^{*}\right)$ states cover an energy window below the top valence level (above bottom conduction level) of about $4 \mathrm{eV}$, while the rest of the electronic structure has $\sigma$ and $\sigma^{*}$ character. Therefore, in the energy range $0-10 \mathrm{eV}$, EELS spectra with $\mathbf{Q}$ along the $c^{*}$ axis (which belongs to the plane of the molecules) have more intense peaks, while for momentum transfers in the $a^{*} b^{*}$ plane solid picene is much less polarizable. For $\mathbf{Q}$ in the $a^{*} b^{*}$ plane, solid picene still behaves as if it were a finite system. Thus the loss function $-\operatorname{Im} \epsilon_{M}^{-1}(\mathbf{Q}, \omega)$, in RPA and in absence of LFE, is approximately proportional to the product between the imaginary part of the independentparticle polarizability $\operatorname{Im} \chi_{0}(\mathbf{Q}, \omega)$ and the Coulomb potential $v(\mathbf{Q})$. The $\mathbf{Q}$ dependence of $\chi_{0}$ can be analyzed in terms of oscillator matrix elements (ME) and the joint density of states (JDOS). For the charge localization on the molecular units, the ME between Bloch wave functions $\rho_{n, n^{\prime}}^{\mathbf{k}}(\mathbf{q}+\mathbf{G})=$ $\left\langle n, \mathbf{k}+\mathbf{q}\left|e^{i(\mathbf{q}+\mathbf{G}) \cdot \mathbf{r}}\right| n^{\prime}, \mathbf{k}\right\rangle$ in a first approximation do not depend on the Bloch wave vector $\mathbf{k}$, so that the ME do not show dispersion in $\mathbf{Q}$. Moreover, since the bandwidths are very small, the JDOS is a constant that does not depend on $\mathbf{Q}$. Thus, in absence of LFE, an increase of the momentum transfer does not cause any dispersion of the features of $\operatorname{Im} \chi_{0}$, but it results only in a progressive reduction of the amplitude of $-\operatorname{Im} \epsilon_{M}^{-1}$, being $v(\mathbf{Q})$ proportional to $1 /|\mathbf{Q}|^{2}$. However, when LFE are included in the calculation, they induce a mixing of electronic transitions, which causes a weak negative dispersion of the peaks, in agreement with the experimental data.

Analogously, also for the momentum transfer along the $c^{*}$ direction and in absence of LFE, the plasmon peak (E) at $6.1 \mathrm{eV}$ [upper panel in Fig. 2(b)] displays a small negative dispersion due to the global reduction of $\operatorname{Im} \epsilon_{M}$ at higher $\mathbf{Q}$. Instead, the (interband) peak (D) shows a remarkable dispersion, which has then to be explained in different terms. In fact, the imaginary part of $\epsilon$ evaluated in absence of LFE presents a small peak at $4.66 \mathrm{eV}$ [see bottom panel in Fig. 2(b)], which is related to $\pi \rightarrow \sigma^{*}$ excitations. For polarizations in the molecular plane, these transitions are forbidden in the dipole approximation (for $\mathbf{Q} \rightarrow 0$ ), but get enhanced when multipole terms are activated by increasing the momentum transfer. The activation of these new transitions determines a splitting of the peak (D), which can be traced back to the rapid oscillation in the real part of $\epsilon$ [see bottom panel in Fig. 2(b)] that the new peak in Im $\epsilon$ induces through the Kramers-Kronig relation. This effect, related to the activation of multipole excitations, is instead not visible for the $a^{*} b^{*}$ polarizations, since in that case the various transitions are already allowed in the dipole limit. Noteworthily, activations of multipole excitations are responsible, for instance, also for the negative plasmon dispersion in a free-electron-like material like $\mathrm{Cs},{ }^{25,26}$ which should be unexpected from the quadratic positive plasmon dispersion of the HEG. Finally, when LFE are included in the calculation of the loss function, both structures (D) and (E) are blueshifted by about $2 \mathrm{eV}$, and mixing of transitions at different energies completely compensate the band-structure effects just discussed for the peak (D). The two features, (D) and (E), do not have a momentum dispersion in the spectrum with LFE [see Fig. 2(a)] and in the experimental results [see Fig. 2(c)]. Thus local fields have opposite effects on the EELS for $\mathbf{Q}$ belonging to the $a^{*} b^{*}$ plane and along 
the $c^{*}$ axis. For the former, LFE are responsible for the small negative dispersion of the features present in the EELS spectra, while for the latter, they completely compensate band-structure effects, resulting in a negligible dispersion.

The analysis performed up to now has shown how the strong localization of the electronic wave functions affects remarkably the EELS spectra of picene resulting in the appearance of structures with negative or almost negligible dispersion. The polarization properties of the molecular units obtained from this analysis will be of fundamental importance also in the rest of the paper to describe a background screening when picene is doped with potassium and becomes metallic.

\section{DOPED PICENE}

The effect of potassium-doping on the EELS of picene is threefold. In fact, comparing the spectra of picene (see Figs. 1 and 2) and $K_{3}$ picene (see Fig. 3) we note that (i) an increase of the bandwidths, due to the larger intermolecular hopping, determines a broadening of all the sharp spectral features discussed so far, and (ii) in addition to the occupation of picene bands that derive from LUMO states, potassium doping also reduces the gap between bands deriving from HOMO and LUMO states of picene, as a consequence of the metallic screening in $\mathrm{K}_{3}$ picene (this is confirmed also by $G W$ band-structure calculations, not shown here ${ }^{27}$ ). Therefore the structures in the EELS of picene are downshifted in $\mathrm{K}_{3}$ picene; (iii) a prominent new peak appears at low energy, in all polarization directions, in the former band gap of pristine picene, as already reported in Ref. 28 by some of us. This peak, which is linked to metallicity in $\mathrm{K}_{3}$ picene, is a signature of a plasmon excitation, as it can be inferred looking at the real and imaginary parts of the dielectric function in Fig. 4(a) $\left(\operatorname{Re} \epsilon_{M}=0\right.$ at the energy of the peak). In the rest of the paper, we will focus on the momentum dispersion of this plasmon excitation.

In order to better understand the nature of this lowenergy plasmon, we evaluated the spectra separating three different contributions (see Fig. 4): (i) taking into account only electronic transitions involving the three metallic $\pi^{*}$ bands and without LFE (green lines), (ii) taking into account also interband transitions (blue lines), and (iii) including LFE (black lines). Comparing the imaginary parts of the dielectric functions calculated with and without interband contributions [see Fig. 4(a)], we find that for $\mathbf{Q}$ in the $a^{*} b^{*}$ plane, the prominent peak is related only to transitions among occupied $\pi^{*}$ bands, so that the plasmon peak in the loss spectra is due to collective oscillations of the conduction electrons. On the contrary, for $\mathbf{Q}$ along the $c^{*}$ axis, interband transitions give a remarkable contribution as well.

The free-electron contribution due to intraband transitions of the six conduction electrons would give rise to a "bare plasmon," which we simulated by considering only transitions between metallic bands. The bare plasmon frequency has a strong negative dispersion along each direction [green lines in Fig 4(b)], a behavior opposite to that of the HEG, and which has been discussed in Ref. 29 on the basis of a tight-binding model. Here, instead, following Aryasetiawan and Karlsson, ${ }^{26}$ we
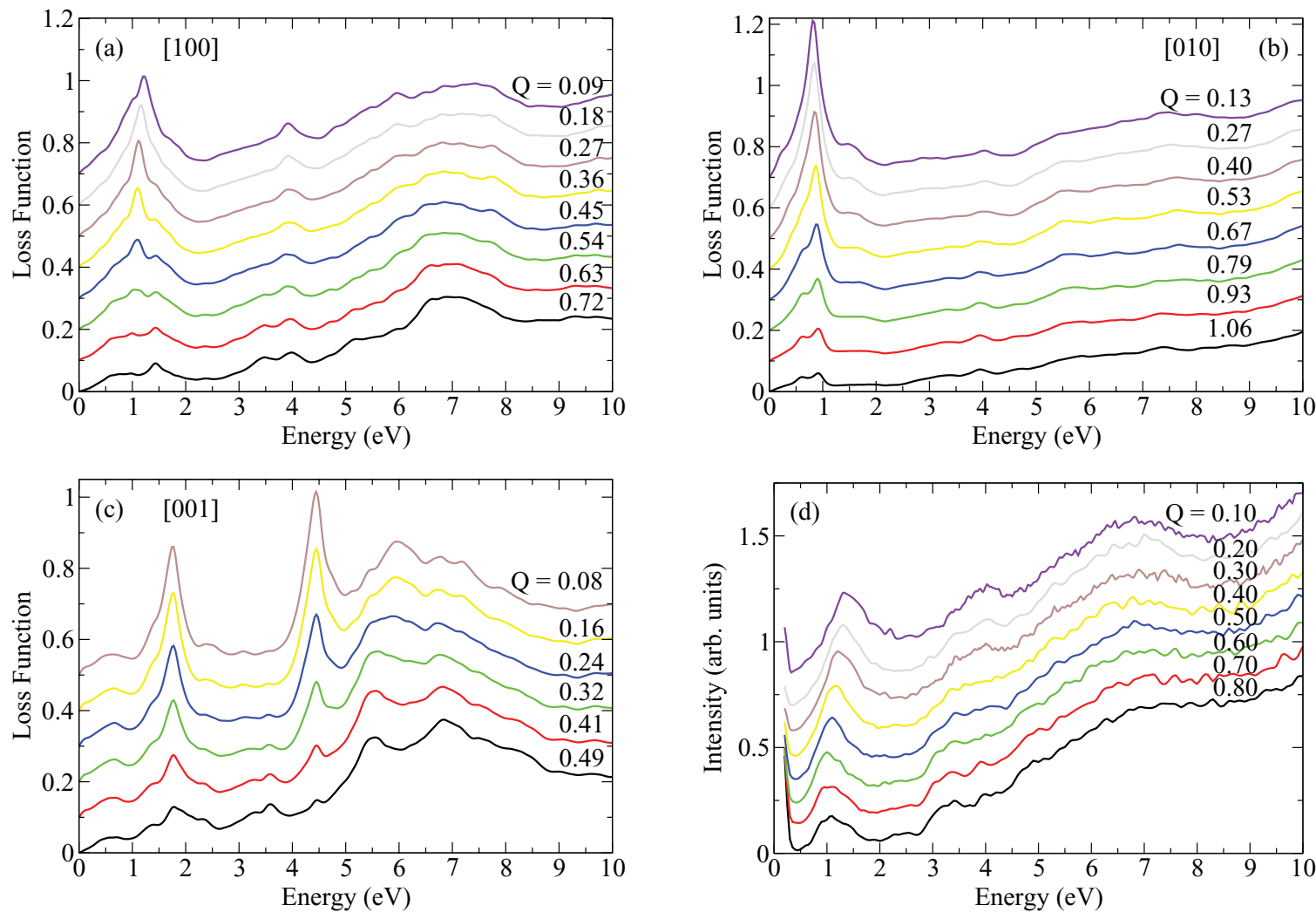

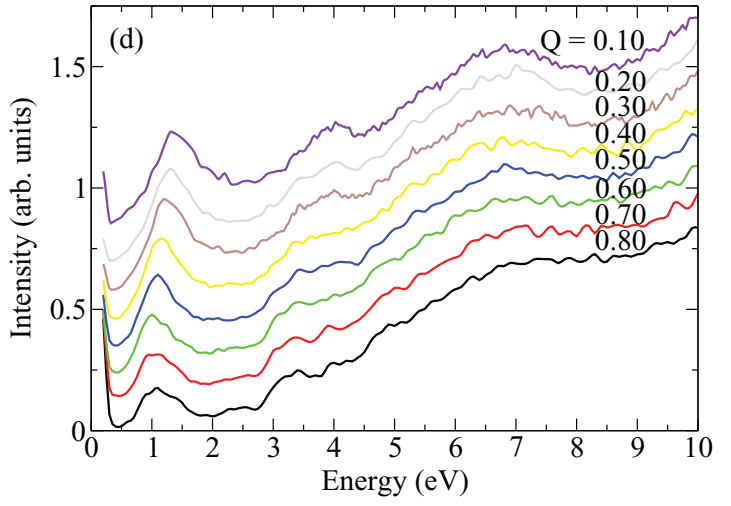

FIG. 3. (Color online) Calculated loss function of $\mathrm{K}_{3}$ picene for momentum transfer $\mathbf{Q}$ (in $\AA^{-1}$ units) along the $a^{*}$ (a), $b^{*}$ (b), and $c^{*}$ axes (c), respectively. (d) Experimental EELS for samples with preferential orientation in the $a b$ plane. 

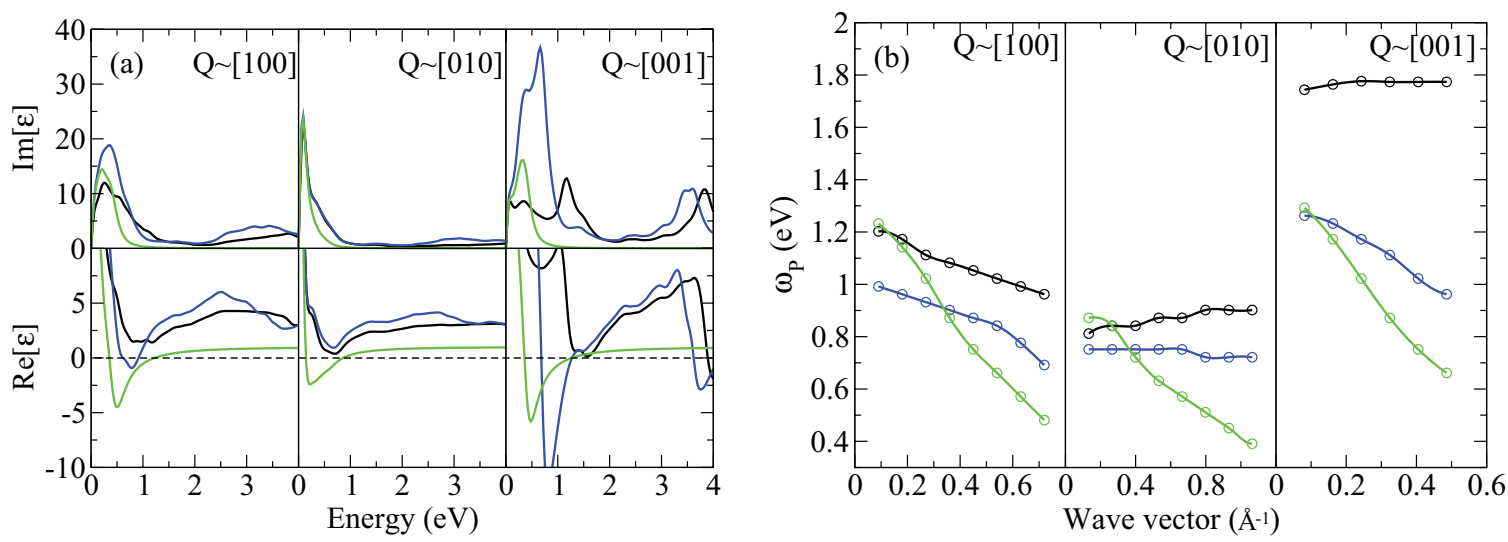

FIG. 4. (Color online) Real and imaginary parts of the dielectric function evaluated at a small wave vector (a) and plasmon dispersion (b) in different approximations: full calculation (black lines), without LFE [blue (dark) lines] and without LFE including only transitions among conduction $\pi^{*}$ bands [green (light) lines].

consider the Kramers-Kronig relations between the imaginary and the real parts of the dielectric function, and define

$$
\begin{aligned}
D(\mathbf{Q}, \omega)= & \frac{2}{\pi}\left[\int_{\omega}^{\infty} d \omega^{\prime} \frac{\omega^{\prime} \operatorname{Im} \epsilon\left(\mathbf{Q}, \omega^{\prime}\right)}{\omega^{\prime 2}-\omega^{2}}\right. \\
& \left.-\int_{0}^{\omega} d \omega^{\prime} \frac{\omega^{\prime} \operatorname{Im} \epsilon\left(\mathbf{Q}, \omega^{\prime}\right)}{\omega^{2}-\omega^{\prime 2}}\right]
\end{aligned}
$$

such that $\operatorname{Re} \epsilon(\mathbf{Q}, \omega)=1+D(\mathbf{Q}, \omega)$. At the plasmon frequencies $\omega=\omega_{p}(\mathbf{Q})$, by definition, $\operatorname{Re} \epsilon\left(\mathbf{Q}, \omega_{p}\right)=0$. Both integrals in Eq. (2) are positive, so they give opposite contributions to $D(\omega)$. Thus, if at a generic $\omega=\bar{\omega}$, the second term (from frequencies $\omega^{\prime}<\bar{\omega}$ ) as a function of $\mathbf{Q}$ increases more (or decreases less) than the first one (for $\omega^{\prime}>\bar{\omega}$ ), then $\operatorname{Re} \epsilon$ at $\omega=\bar{\omega}$ decreases with $\mathbf{Q}$. Since $\operatorname{Re} \epsilon$ has a positive slope at the plasmon frequency $\omega=\omega_{p}$, this implies that a crossing of the zero axis by $\operatorname{Re} \epsilon$ then would take place at a larger $\omega_{p}$.

In absence of interband transitions, we have only contributions at $\omega$ smaller than $\omega_{p}$, so only from the second term in Eq. (2). Therefore, for a positive dispersion of the plasmon frequency, the second integral in Eq. (2) must be an increasing function of $\mathbf{Q}$. Even when an increase of $\mathbf{Q}$ causes a lowering of $\operatorname{Im} \epsilon(\mathbf{Q}, \omega)$, if the features in $\operatorname{Im} \epsilon(\mathbf{Q}, \omega)$ have a positive dispersion, like in the HEG, the integral in Eq. (2) can increase, causing a positive dispersion for $\omega_{p}$. However, this is not the case of picene since, as discussed previously, $\operatorname{Im} \epsilon(\mathbf{Q}, \omega)$ does not disperse, due to the strong localization of the wave functions on the molecules. This results in the strong negative dispersion of the bare plasmon. Including the contributions of interband transitions at frequencies higher than $\omega_{p}$ [first term in Eq. (2)] reduces the negative dispersion of the bare plasmon, as demonstrated by comparing green and blue lines in Fig. 4(b) (for $\mathbf{Q}$ along $c^{*}$ the plasmon involves partially also some interband transitions, so that the plasmon dispersion remains negative even including their contribution). In fact, as a response to the collective oscillations of the conduction electrons, the single molecules polarize, screening the bare plasmon. This background screening is, in a first approximation, given by the interband dielectric constant $\epsilon_{b}(\mathbf{Q})$, which describes how the molecules polarize. Since $\epsilon_{b}(\mathbf{Q})$ is a decreasing function of $\mathbf{Q}$, interband transitions reduce the strength of the negative dispersion. Finally, LFE are responsible for mixing among intraband and higher energy interband $\pi \rightarrow \pi^{*}$ transitions. LFE become more important as $\mathbf{Q}$ increases, since in real space this implies probing induced charge densities on a shorter scale, where charge inhomogeneities become more relevant. Therefore LFE further reduce the negative plasmon dispersion, as we find comparing blue and black lines in Fig. 4(b).

On a quantitative level, the effect of interband transitions and crystal local fields on the plasmon dispersion depends on the polarization properties of the molecules. These properties have been obtained in the analysis of the loss functions of pristine picene. For $\mathbf{Q}$ along $c^{*}$, the molecules are highly polarizable, so that the corrections to the bare plasmon are large and the negative dispersion is completely suppressed: $\omega_{p}$ presents a small positive dispersion. On the contrary, for $\mathbf{Q}$ along the $a^{*}$ axis, the effect is weaker (the molecules are less polarizable) and the negative dispersion of the bare plasmon is only reduced, but still visible, as we can see also in the experimental spectra in Fig. 3(d).

\section{CONCLUSIONS}

In conclusion, from the previous analysis of the electron energy loss spectra, we can infer a general picture of the plasmon dispersion common to all doped molecular crystals, which are characterized by the competition between charge localization and metallicity. In fact, a negligible plasmon dispersion has been reported also in alkali-doped $\mathrm{C}_{60}$ compounds. ${ }^{30}$ Since the electrons are mainly confined to the individual molecules, matrix elements and joint density of states do not disperse as a function $\mathbf{Q}$, so that the bare frequency associated to the plasma oscillations of the conduction electrons has a negative dispersion. This effect is contrasted by interband transitions (through screening effects) and local-field effects, which depend on the ability of the molecules to polarize along the different directions. We expect that these polarization effects, which reduce the negative dispersion, become more important as the dimension of the molecules increases, so that the negative plasmon dispersion should be stronger as the number of benzene rings in the molecular units is reduced. 
Finally, the good comparison between theoretical and experimental data shows that electronic correlation in doped picene is adequately treated at the level of the random-phase approximation, in seeming contrast to the hypothesis that doped picene is a strongly correlated superconductor.

\section{ACKNOWLEDGMENTS}

We thank T. Miyake (author of Ref. 16) for providing us with the relaxed structure of doped picene. This work has been supported by the Deutsche Forschungsgemeinschaft
(Grant Nos. KN393/13 and KN393/14). We acknowledge financial support also from Spanish MEC (FIS201165702-C02-01), ACI-Promociona (ACI2009-1036), Grupos Consolidados UPV/EHU del Gobierno Vasco (IT-319-07), and the European Research Council Advanced Grant DYNamo (ERC-2010-AdG Proposal No. 267374), and computational time from Barcelona Supercomputing Center, "Red Espanola de Supercomputacion", SGIker ARINA (UPV/EHU). We thank X. Andrade and J. Alberdi Rodriguez for the efficient computer-cluster installation in San Sebastian.
${ }^{1}$ S. R. Forrest, Nature (London) 428, 911 (2004).

${ }^{2}$ M. E. Gershenson, V. Podzorov, and A. F. Morpurgo, Rev. Mod. Phys. 78, 973 (2006).

${ }^{3}$ M. A. Baldo, D. F. O'Brien, Y. You, A. Shoustikov, S. Sibley, M. E. Thompson, and S. R. Forrest, Nature (London) 395, 151 (1998).

${ }^{4}$ R. H. Friend, R. W. Gymer, A. B. Holmes, J. H. Burroughes, R. N. Marks, C. Taliani, D. D. C. Bradley, D. A. Dos Santos, J. L. Brédas, M. Lögdlund, and W. R. Salaneck, Nature (London) 397, 121 (1999).

${ }^{5}$ P. Peumans, S. Uchida, and S. R. Forrest, Nature (London) 425, 158 (2003).

${ }^{6}$ G. Li, V. Shrotriya, J. Huang, Y. Yao, T. Moriarty, K. Emery, and Y. Yang, Nat. Mater. 4, 864 (2005).

${ }^{7}$ A. F. Hebard, M. J. Rosseinsky, R. C. Haddon, D. W. Murphy, S. H. Glarum, T. T. M. Palstra, A. P. Ramirez, and A. R. Kortan, Nature (London) 350, 600 (1991).

${ }^{8}$ K. Tanigaki, T. W. Ebbesen, S. Saito, J. Mizuki, J. S. Tsai, Y. Kubo, and S. Kuroshima, Nature (London) 352, 222 (1991).

${ }^{9}$ O. Gunnarsson, Rev. Mod. Phys. 69, 575 (1997).

${ }^{10}$ R. Mitsuhashi, Y. Suzuki, Y. Yamanari, H. Mitamura, T. Kambe, N. Ikeda, H. Okamoto, A. Fujiwara, M. Yamaji, N. Kawasaki, Y. Maniwa, and Y. Kubozono, Nature (London) 464, 76 (2010).

${ }^{11}$ X. F. Wang, R. H. Liu, Z. Gui, Y. L. Xie, Y. J. Yan, J. J. Ying, X. G. Luo, and X. H. Chen, e-print arXiv:1102.4075v1 (unpublished).

${ }^{12}$ Y. Kubozono, M. Mitamura, X. Lee, X. He, Y. Yamanari, Y. Takahashi, Y. Suzuki, Y. Kaji, R. Eguchi, K. Akaike, T. Kambe, H. Okamoto, A. Fujiwara, T. Kato, T. Kosugi, and H. Aoki, Phys Chem Chem Phys 13, 16476 (2011).

${ }^{13}$ F. Ortmann, F. Bechstedt, and K. Hannewald, New J. Phys. 12, 023011 (2010).

${ }^{14}$ M. Capone, M. Fabrizio, C. Castellani, and E. Tosatti, Rev. Mod. Phys. 81, 943 (2009).

${ }^{15}$ F. Roth, M. Gatti, P. Cudazzo, M. Grobosch, B. Mahns, B. Büchner, A. Rubio, and M. Knupfer, New J. Phys. 12, 103036 (2010).
${ }^{16}$ T. Kosugi, T. Miyake, S. Ishibashi, R. Arita, and H. Aoki, J. Phys. Soc. Jpn. 78, 113704 (2009).

${ }^{17}$ H. Okazaki, T. Wakita, T. Muro, Y. Kaji, X. Lee, H. Mitamura, N. Kawasaki, Y. Kubozono, Y. Yamanari, T. Kambe, T. Kato, M. Hirai, Y. Muraoka, and T. Yokoya, Phys. Rev. B 82, 195114 (2010).

${ }^{18}$ A. De, R. Ghosh, S. Roychowdhury, and P. Roychowdhury, Acta Crystallogr. C 41, 907 (1985).

${ }^{19}$ J. Fink, Adv. Electron. Electron Phys. 75, 121 (1989).

${ }^{20}$ S. L. Adler, Phys. Rev. 126, 413 (1962).

${ }^{21}$ N. Wiser, Phys. Rev. 129, 62 (1963).

${ }^{22}$ P. Giannozzi, S. Baroni, N. Bonini, M. Calandra, R. Car, C. Cavazzoni, D. Ceresoli, G. L. Chiarotti, M. Cococcioni, I. Dabo, A. Dal Corso, S. Fabris, G. Fratesi, S. de Gironcoli, R. Gebauer, U. Gerstmann, C. Gougoussis, A. Kokalj, M. Lazzeri, L. Martin-Samos, N. Marzari, F. Mauri, R. Mazzarello, S. Paolini, A. Pasquarello, L. Paulatto, C. Sbraccia, S. Scandolo, G. Sclauzero, A. P. Seitsonen, A. Smogunov, P. Umari, and R. M. Wentzcovitch, J. Phys. Condens. Matter 21, 395502 (2009).

${ }^{23}$ A. Marini, C. Hogan, M. Grüning, and D. Varsano, Comp. Phys. Commun. 180, 1392 (2009).

${ }^{24}$ P. L. de Andres, A. Guijarro, and J. A. Verges, Phys. Rev. B 83, 245113 (2011)

${ }^{25}$ A. vom Felde, J. Sprosser-Prou, and J. Fink, Phys. Rev. B 40, 10181 (1989).

${ }^{26}$ F. Aryasetiawan and K. Karlsson, Phys. Rev. Lett. 73, 1679 (1994).

${ }^{27}$ Notice that while for picene, $G W$ corrections and the excitonic binding energy are both sizable and do not compensate each other, ${ }^{15}$ in $\mathrm{K}_{3}$ picene, $G W$ corrections do not modify significantly the LDA results and, for the metallic screening of electron-hole interactions, we do not expect bound excitons.

${ }^{28}$ F. Roth, B. Mahns, B. Büchner, and M. Knupfer, Phys. Rev. B 83, 144501 (2011).

${ }^{29}$ V. V. Kresin and V. Z. Kresin, Phys. Rev. B 49, 2715 (1994).

${ }^{30}$ O. Gunnarsson, V. Eyert, M. Knupfer, J. Fink, and J. F. Armbruste, J. Phys. Condens. Matter 8, 2557 (1996). 